\title{
The Effects of Life Line Facial Cream/Periobitum Serum and Micro-Needling of Photodamaged Skin: A Split-Face Randomized Study
}

\author{
Gordon H. Sasaki \\ Department of Plastic Surgery, Loma Linda University Medical Center, Loma Linda, USA \\ Email: ghsasakimd@drsasaki.com
}

Received 26 September 2015; accepted 14 October 2015; published 19 October 2015

Copyright (C) 2015 by author and OALib.

This work is licensed under the Creative Commons Attribution International License (CC BY). http://creativecommons.org/licenses/by/4.0/

(c) (i) Open Access

\section{Abstract}

Background: Human growth factors serve as secretomes that regulate skin cell proliferation/differentiation and production of extracellular elements of the matrix and treat photo-aging. Microneedling of skin can also improve aging skin by facilitating normal wound healing of the temporary dermal conduits for therapeutic and cosmetic benefits. Objectives: This open, randomized split-face and single-center controlled study evaluated the safety and efficacy of the application of a cosmeceutical formulation, which incorporated growth factors derived from cultured pluripotential stem cells of unfertilized human ovum, in the treatment of photo-damaged facial skin. The use of micro-needling to stem cell-treated skin was evaluated for additional benefit. Material and Methods: Ten subjects in Group 1 with mild-to-moderate photo-damaged skin received the growth factor emulsion to one side of the face and serum to the eyelids/periorbitum and placebo on the contralateral side for twelve (12) weeks. Ten subjects in a separate Group 2 with similar photodamaged skin received the same growth factor emulsions and serum to both sides of the face and eyelids/periorbitum, but only one side was treated with four micro-needling procedures four weeks apart. Subjects in Group 1 were assessed at baseline, 6 weeks and 12 weeks with the Canfield VISIA Investigator System and standardized 2D photography. Subjects in Group 2 were similarly assessed with the addition of the DermaLab Cortex System. Data analysis compared the p-values with the alpha level for statistical difference. Results: Group 1 subjects demonstrated a beneficial effect by VISIA Analysis on reduction of pigmented spots, UV-light damage with more effective effacement of wrinkles and textural changes after three months compared with that observed on the placebo side. Group 2 subjects demonstrated more improvement by VISIA Analysis in reduction of pigmentations, UV-light damage, porphyrin spots, and wrinkles with more textural smoothness with combined treatments than with cosmeceutical application alone. Cortex Analysis of skin, receiving combined treatments, demonstrated marked improvements in skin elasticity and hydration observed after cosmeceutical application alone. Conclusions: The used growth factor-con- 
taining creams and serum have a salutary effect on photodamaged skin whose effects may be further enhanced with the addition of micro-needling without introduction of side-effects or complications. Further investigations with a larger cohort of subjects for longer treatment periods will be needed to confirm and extend these initial findings.

\title{
Keywords
}

\author{
Human Growth Factor Cream and Serum, Micro-Needling, Skin Photo-Aging, Safety and Efficacy
}

\author{
Subject Areas: Dermatology, Drugs \& Devices, Surgery \& Surgical Specialties
}

\section{Introduction}

The skin's visco-mechanical properties, protective cells and structures, and restorative capabilities diminish as a consequence of genetic influences and multiple environmental factors. Patients assess their aging skin often in terms of skin thinning, fragility, loss of plumpness, reduced clarity and luster, impaired hydration, pigmentations, vascular clusters, and wrinkles. These manifestations are believed to be a consequence of impaired functioning of keratinocytes, melanocytes and immunocompetent cells within the epidermis, as well as fibroblasts' impairment to regulate collagen, elastin and glucosoaminoglycan matrix stability within the dermis. Skin has the ability to repair epidermal and dermal damages from photo- and chronological aging using the body's natural mechanisms that include the use of growth factors. A skin care product has recently been developed that introduces a human non-embryonic pluripotent stem cell-based serum with growth factors that regulate specific cellular activities such as cell proliferation, repair damaged DNA, increase collagen and elastin formation, and replenish the extracellular matrix [1]. Through proprietary liposome technology, released growth factors penetrate the stratum corneum to reverse cell dysfunction, help repair UV damage and protect the skin from daily assaults. Micro-needling of skin [2] disrupts and bypasses the formidable barrier of the stratum corneum by delivering numerous micro-channels into the dermis, thereby forming conduits for wound healing and as an effective delivery system for macromolecules within cosmeceuticals [3] [4]. The purpose of this clinical half-face study is to determine the safety and efficacy of this proprietary product and whether the addition of micro-needling can further improve results.

\section{Materials and Methods}

\subsection{Study Design}

This was an open, randomized single-center, split-face controlled study that 1) compared the safety and efficacy of proprietary growth factors versus a non-cosmeceutical moisturizer to improve the aged skin, and 2) determined whether the combined treatment of micro-needling procedures and growth factors could increase the benefits observed only from the use of growth factors contained within the same skin care product line. Baseline investigator evaluations, along with standardized digital photography, Canfield Investigator's Skin Care Absolute Score Analyses (pigmented spots, wrinkles, texture, pores, UV spots, and porphyrin), and DermaLabCortex Measurements (elasticity, trans-epidermal water loss [TEWL], hydration, pigmentation and erythema) were determined thereafter at six and twelve weeks of treatment for assessment of parameters.

\subsection{Study Population}

Of over 100 subjects evaluated in the author's clinical practice, only 20 healthy subjects were qualified and provided informed consents for participation either in Protocol \#1 (10 subjects, half-face treatment with growth factor skin care versus placebo-control) or in Protocol \#2 (10 subjects, half-face treatments with micro-needling and growth factor skin care versus growth factor skin care). Included females and males were limited between 35 - 65 years of age, self-reported Fitzpatrick Skin Types I to IV demonstrating periocular and perioral wrinkling, including facial pigmented spots, textural changes, enlarged pores and skin laxity. Participants were will- 
ing 1) to use a supplied SPF 35 product, abstain from extended periods of facial sun exposure and all uses of artificial tanning for the duration of the study, 2) to continue use of all regular brands of color cosmetics without claims of anti-aging effects, and 3) to refrain from any facial treatments such as medical skin care. Subjects had no pre-existing skin conditions, such as active infections, autoimmune diseases, known untreated skin cancers, or uncontrolled diabetes mellitus. Exclusion criteria included the following: unwillingness to avoid excess sunlight or use of sunscreen, unwillingness to forgo any other topical dermatological therapy as well as use of $\alpha$ and $\beta$-hydroxy acids, retinoids, or vitamin C-, E-, D-containing topicals, within six months before as well as throughout the course of the study. Washout periods adhered to by subjects included the following: 6 months free from dermabrasion, micro-needling, deep chemical peels, ablative and non-ablative skin treatments, neurotoxin and filler injections, skin tightening procedures, and facial surgery. No subject may be pregnant, breastfeeding, or planning a pregnancy during the study. No subject could regularly use anti-inflammatories or antihistamines or have any history of sensitivity to skin treatment products. Prior to inclusion, subjects gave their written informed consent in accordance with the ethical requirements for a cosmetic study. This study was conducted in accordance with the International Conference of Harmonization, Tripartite Guideline on Good Clinical Practice, and standard practices of BioScreen Clinical Services.

\subsection{Skin Care Products}

The growth factors (Daily Defense Complex; Lifeline Skin Care Inc., Carlsbad, CA) in this study were derived from cultured pluripotent stem cells obtained by parthenogenesis from an unfertilized human egg. The Lifeline growth factors from human stem cells contain 1) potent epidermal growth factors that influence cellular proliferation, differentiation, and survival, 2) fibroblast growth factors that speed wound repair, and 3) keratinocyte growth factors that speed cellular division. These growth factors are believed to work together to create and maintain health skin. The emulsion also contained water, caprylic/capric triglyceride, stearic acid, ButyrospermumParkii (shea) butter, glycerin, cetyl alcohol, glyceryl stearate, PEG-100 stearate, algae extract, arganiaspinosa spout cell extract, helianthus annuus (sunflower) seed oil, rosmarinusofficinalis (rosemary) leaf extract, saccharomyces cerevisae extract, collagen amino acids, micrococcus lysate, phospholipids, isomalt, cetearyl alcohol, urea, glucosamine $\mathrm{HCl}$, ceteareth-20, niacinamide, carbomer, tocopheryl acetate, polyvinylalcoholcrosspolymer, retinylpalmitate, sodium PCA, sodium starch octenylsuccinate, calcium pantothenate, disodium EDTA, maltodextrin, panthenol, sodium ascorbyl phosphate, lecithin, ethyl ferulate, polyglycery $1-5$, trioleate, pyridoxine $\mathrm{HCl}$, ubiquinone, sodium benzoate, silica, nylon-12 fluorescent brightener 230 salt, hexylene glycol, disodium uridine phosphate, aminomethyl propanol, ethylhexyglycerin, caprylyl glycol, phenoxyethanol, and citric acid.

The Lifeline Recovery Night Moisture Serum in this study contained similar growth factors derived from human stem cells and includes B complex nutrients, green tea extract, hyaluronic acid, amino acids/peptides and safflower seed oil. The B Complex Nutrients are believed to increase microcirculation to the skin. The Green Tea Extract is thought to act as an anti-oxidant that minimizes free radical damage from sun and environmental injury. Hyaluronic Acid is believed to retain skin moisture and aid in the integrity of the skin's structural components, collagen and elastin. The Amino Acids and Peptides are thought to stimulate the production of hyaluronic acids, collagen and elastin. The safflower Seed Oil contains the essential fatty acid, linoleic acid, which contributes to the formation of the moisture barrier within the epidermis.

The Lifeline Eye Firming Complex in this study contained similar growth factors derived from human stem cells. deionized water, glycerin, ethylhexylpalmitate, cetearyl alcohol, cocosnucifera (coconut) oil, caprylic/capric triglyceride, caffeine, butyrospermumparkii (shea butter) fruit, cetearyolivate, sodium starch, octenylsuccinate, myristyl alcohol, glycosaminoglycans, palmitolyoligopeptide, palmitoyl tetrapeptide-7, hydrolyzed jojoba esters, chamommillarecutita (matricaria) flower extract, sodium hyaluronate, oleaeuopaea (olive), fruit oil, aloe barbadensis leaf extract, tocopheryl acetate, tetrahexyldecylascorbate, sodium polystyrene sulfonate, sorghum bicolor stalk juice, anthemisnobilis flower oil, sorbitanolivate, zea may (corn) starch, hydrolyzed corn starch, beeswax, hydrolyzed corn starch octenylsuccinate, myristylglucoside, polyquarternium-67, ceteraryglucoside, silica, butylene glycol, carbomer, polysorbate 20 , hydroxyethylbehenamidopropyldimonium chloride, polysorbate 60 , acrylates/C10-30 alkyl acrylate crosspolymer, hexylene glycol, ammonium acryloyldimethyltaurate/VP copolymer, phenoxyethanol, sodium hydroxide, caprylyl glycol, and ethylhexyl glycerin. The placebo (Neutro- 
gena Oil-Free Moisture, SPF 35) in this study contained avobenzone $3 \%$, homosalate $12 \%$, octisalate $5 \%$, octocrylene $2.35 \%$, oxybenzone $6 \%$, water, dimethicone, trisiloxane, diethylhexyl 2, 6-naphthalate, glycerin, potassium cetyl phosphate, glyceryl stearate, PEG-100 stearate, ceterayl alcohol, behenyl alcohol, caprylylmethicone, styrene/acrylates copolymer, ethylhexylglycerin, benzyl alcohol, methylparaben, cetearylglucoside, xanthan gum, alcohol, methylparaben, ceterarylglucoside, xanthan gum, propylparaben, disodium EDTA, BHT, and methylisothiazolinone. The facial cleanser (Neutrogena Extra Gentle Cleanser) in this study contained water purified, glycerin, caprylic/capric triglycerides, cetylricinoleate (chamomile extract0, isohexadecane, TEA cocyl glutamate, methyl glucose sesquisterarate, stearyl alcohol, cetyl alcohol, PEG 40 hydrogenated castor oil, PEG 20 methyl glucose sesquitearate, bisabolol (anti-irritant and anti-inflammatory), chamomile extract, tocopherylacetate (vitamin E), dipotassiumglycyrrhizate (licorice root extract), stearylglycyrrhetinate, tetrasodium EDTA, acrylates/C10 30 alkyl acrylate crosspolymer, triethanolamine, BHT, geranium, dipropylene glycol, phenoxyethanol, propylparaben, and methylparaben.

\subsection{Devices}

The micro-needling device (DermaPen, LLC, Salt Lake City, UT) had an input power of $5 \mathrm{~V}$ with an adapter that ranged from AC 100-240V, 50-50HZ. Sterilized single-use disposable, spring-loaded micro-needling tips contained 11 needles which were 33 gauge and adjusted to a $0.5 \mathrm{~mm}$ depth setting at the recommended manufacture speed between 4 and 5 settings. Three of six skin care specialists with over three year experience in the author's medical skin care center performed the micro-needling for uniformity and consistency of the procedure.

The DermaLabSkinLab Combo Module with Cortex Technology (Hadsund, Denmark) is an established device to measure a number of skin parameters with individual probes. The device was calibrated by the manufacturer with a Declaration of Conformity prior to initiation of this study. The subjects were acclimated to the ambient environment for a period of thirty minutes prior to evaluations. The areas of involvement were marked on the facial surface using a standard template to ensure that the instruments were repositioned in the same place at each visit. By a suction method, an Elasticity Vacuum Probe measured the ViscoElasticity (VE) properties of both the elevation retraction phases of a mechanical stress (MPa: mega Pascal units) applied to baseline and treated skin at selected location on the skin surface of the face. The TEWL Probe measured the water loss through designated spot of facial skin with two sets of sensors (temperature and humidity) mounted in its chamber. The TEWL result was shown in $\mathrm{g} / \mathrm{m}^{2} / \mathrm{hr}$ as a function of time as the mean value over the last 5 seconds. By eight contact pins, the Hydration Moisture Probe measured the conducting properties of the upper layers of the selected skin location that was subjected to an alternating voltage. The conductance measurement was expressed in the unit of micro-Siemens ( $\mu \mathrm{S})$. The Skin Color Probe of melanin (pigmentation) and erythema was based on an active color detecting chip illuminated by two high intensity white LED's during positioning of the probe on a designated facial skin area. Two technicians, experienced in the Cortex technology, performed all DermaLab skin measurements at the same location at baseline and treatment intervals.

The VISIAS Skin Analysis System (CANFIELD Imaging Systems, Fairfield, NJ) consisted of an imaging booth and VISIA software that captured comparison features over time such pigment spots, periocular and perioral wrinkles, texture skin color and smoothness, pores size, UV light spots and porphyrin bacterial excretions. The viewing analysis results were displayed as absolute scores that tracked the intensity of the feature being analyzed as an indicator of treatment effectiveness.

\subsection{Method}

Each subject's treatment regimen in Protocol \#1 included daily morning use of the Neutrogenia facial cleanser, followed by randomized split-face application of Lifeline Daily Defense Complex (face) and Eye Firming Complex (eyelids and periorbitum), with the contralateral eyelids and face receiving the Neutrogena Oil-Free Moisture SPF 35. Additional SPF 35 sunscreen was applied over the Lifeline complexes. The evening regimen included the use of the Neutrogena facial cleanser, followed by the application of Lifeline Night Moisture Serum (face) and Eye Firming Complex (eyelids and periorbitum) to the active side, with the contralateral inactive side receiving the Neutrogena Oil-Free Moisturizer. This day and night regimen with the Lifeline products was maintained for 12 weeks. At the end of the study, subjects were offered the same Lifeline products to treat their entire face for an additional three months to balance out the final results. 
Each subject's treatment regimen in Protocol \#2 included four micro-needling procedures separated four weeks apart to the specified half of face. At micro-needling, the designated half face skin was cleansed with the Neutrogenia facial cleanser. The skin was anesthetized with a topical anesthetic EMLA cream (2.5\% lidocaine; $2.5 \%$ prilocaine) for approximately ten minutes under a wrap. The cream was then gently removed with Hibiclens and sterile water. Micro-needling was performed in ten horizontal, ten vertical and ten oblique directions at a setting of $1.0 \mathrm{~mm}$ to half forehead, face, and perioral skin (upper/lower lip and chin). The entire upper/lower eyelids and crow's feet were treated at a setting of $0.5 \mathrm{~mm}$ with five multi-directional passes. After cleansing the skin with sterile saline, the Lifeline Daily Defense Complex and Eye Firming Complex were massaged to the entire face. Additional SPF 35 sunscreen was applied over the Lifeline skin care products. The evening regimen included the use of the Neutrogena facial cleanser, followed by the application of Lifeline Night Moisture Serum (face) and Eye Firming Complex (eyelids and periorbitum) to the entire face. This day and night regimen with the Lifeline products was maintained for 12 weeks. At the end of the study, subjects were offered micro-needling to the entire face every four weeks for three months to even out the final results.

Two independent technicians performed the Canfield VISIA Investigator's Skin Absolute Score Analyses, and standardized 2D photography at baseline, six weeks and three months on all subjects in Protocol \#1 and \#2. The Derma Lab Cortex Analyses was performed by the same technicians only for subjects in Protocol \#2 because the device was unavailable during the treatment of subjects in Protocol \#1. The office statistician performed the calculation of data.

\subsection{Data Analysis}

The data analysis compared the p-value with the alpha level to determine whether the observed data was statistically different. A p-value of $\leq 0.05$ was considered statistically significant.

\section{Results}

Ten subjects were enrolled in Protocol \#1 and in Protocol \#2 and included in the final data analysis.

The participants in each protocol exhibited no significant differences in the mean age/range, Fitzpatrick Skin Types, distribution of gender, except for the number of different ethnicities enrolled in the two study sets (Table 1).

\subsection{Protocol \#1}

VISIA Analysis of absolute values, expressed as a percent change from baseline values, showed a greater average difference in reduction of pigmentation spots $(12.7 \% \pm 9.2 \%$ vs. $1.0 \% \pm 5.4 \%)$ and UV lightspots $(40.1 \% \pm$ $20.1 \%$ vs. $29.0 \% \pm 19.1 \%$ ) in Figure 1 and greater average difference in improvement of wrinkle effacement

Table 1. Demographics of Protocol \#1 and Protocol \#2 that include distribution of number of subjects, average age, age range, Fitzpatrick Skin Type scale, gender and ethnicity.

\begin{tabular}{|c|c|c|c|c|c|c|c|c|}
\hline \multirow{2}{*}{ Lifeline/Placebo } & \multirow{2}{*}{ Age } & \multicolumn{3}{|c|}{ Fitzpatrick Scale } & \multicolumn{2}{|c|}{ Gender } & \multicolumn{2}{|c|}{ Ethnicity } \\
\hline & & II & III & IV & Female & Male & Caucasian & Hispanic \\
\hline No. Pts & 10 & 2 & 3 & 5 & 8 & 2 & 2 & 8 \\
\hline Average Age & 53 & $20 \%$ & $30 \%$ & $50 \%$ & $80 \%$ & $20 \%$ & $80 \%$ & $20 \%$ \\
\hline Age Range & $31-79$ & & & & & & & \\
\hline \multirow{2}{*}{$\begin{array}{l}\text { Lifeline/Lifeline } \\
\text { and Mirconeedling }\end{array}$} & \multirow{2}{*}{ Age } & \multicolumn{3}{|c|}{ Fitzpatrick Scale } & \multicolumn{2}{|c|}{ Gender } & \multicolumn{2}{|c|}{ Ethnicity } \\
\hline & & II & III & IV & Female & Male & Caucasian & Hispanic \\
\hline No. Pts & 10 & 3 & 3 & 4 & 8 & 2 & 5 & 5 \\
\hline Average Age & 53.1 & $30 \%$ & $30 \%$ & $40 \%$ & $80 \%$ & $20 \%$ & $50 \%$ & $50 \%$ \\
\hline Age Range & $44-74$ & & & & & & & \\
\hline
\end{tabular}


Placebo versus Lifeline (VISIA Analysis)
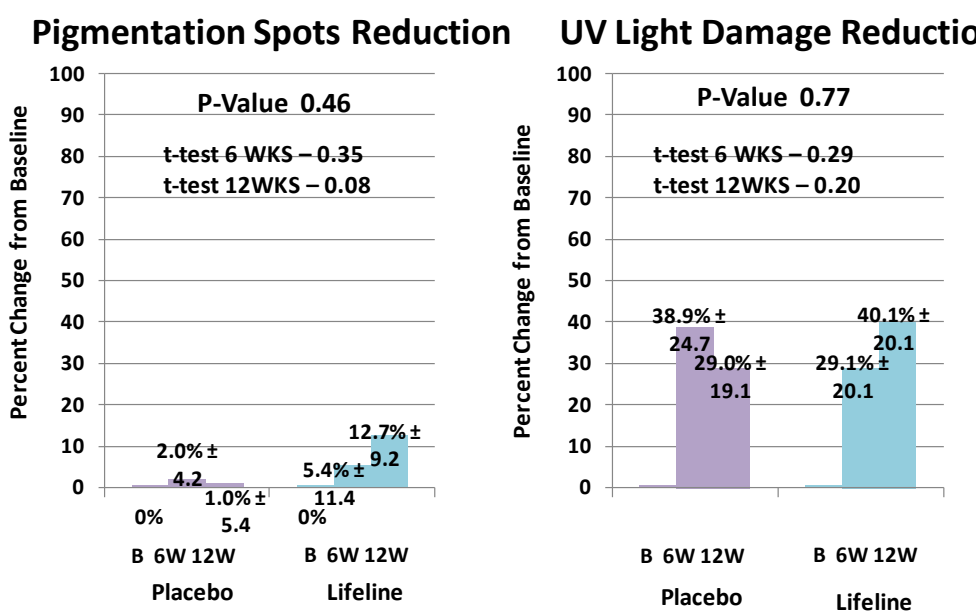

Fugure 1. VISIA Analysis of average percent change of absolute values from baseline values (percent $\pm \mathrm{SD}$ ) in reduction in pigmentation and UV light damage on placebo vs. Lifeline sides of the face at six and twelve weeks.

$(47.2 \% \pm 28.8 \%$ vs. $20.3 \% \pm 21.6 \%)$ and texture smoothness $(32.3 \% \pm 27.1 \%$ vs. $22.1 \% \pm 23.6 \%)$ in Figure 2 on the Lifeline side versus the placebo side of each subject's face at twelve weeks of treatment. At twelve weeks, there was minimal average reduction in pore size spots $(25.4 \% \pm 15.3 \%$ vs. $21.2 \% \pm 14.3 \%)$ and porphyrin reduction $(65.8 \% \pm 33.2 \%$ vs. $68.2 \% \pm 37.6 \%)$ in Figure 3 on the Lifeline side as compared to the placebo side in each of the treated subjects.

\subsection{Protocol \#2}

VISIA Analysis of absolute values, expressed as percent change from baseline values, demonstrated a greater average difference in reduction of pigmentation spots $(6.9 \% \pm 2.1 \%$ vs. $2.1 \% \pm 1.4 \%)$, UV light spots $(29.4 \% \pm$ $23.8 \%$ vs. $20.3 \% \pm 24.0 \%)$ and porphyrin spots $(71.2 \% \pm 33.0 \%$ vs. $60.5 \% \pm 32.4 \%)$ in Figure 4 and greater average difference in improvement of wrinkle effacement $(35.2 \% \pm 24.2 \%$ vs. $20.7 \% \pm 20.9 \%)$ and texture smoothness $(22.6 \% \pm 14.6 \%$ vs. $11.5 \% \pm 11.8 \%)$ in Figure 5 on the Lifeline/micro-needling side than on the Lifeline side at twelve weeks. At twelve weeks, there was a slight average reduction in pore size spots $(20.8 \% \pm$ $23.6 \%$ vs. $16.1 \% \pm 25.9 \%$ ) in Figure 6 on both the Lifeline/micro-needling and the Lifeline side.

Cortex Analysis recorded a greater average difference in improvement of skin elasticity $(60.1 \% \pm 28.9 \%$ vs. $39.5 \% \pm 24.1 \mathrm{MPa}$ units) and hydration $(36.1 \% \pm 23.7 \%$ vs. $28.3 \% \pm 10.7 \mu \mathrm{S}$ units $)$ on the Lifeline/microneedling side than on the Lifeline side at twelve weeks in Figure 7. At twelve weeks, there were minimal average changes in TEWL $\left(23.3 \% \pm 19.9 \%\right.$ vs. $\left.24.0 \% \pm 19.3 \mathrm{~g} / \mathrm{m}^{2} / \mathrm{hr}\right)$, pigment reduction $6.7 \% \pm 5.1 \%$ vs. $7.8 \% \pm$ $3.4 \%)$, and erythema reduction $(7.0 \% \pm 6.9 \%$ vs. $10.0 \% \pm 9.5 \%)$ at twelve weeks on the Lifeline/micro-needling side compared to the Lifeline side of the face in Figure 8.

\section{Discussion}

Results from this placebo-controlled, split-face study corroborated observations from previous open-label studies using the same Lifeline products (Defensive Day Moisture Serum, Recovery Night Moisture Serum, Eye Firming Complex) that reported a reduction of fine lines and wrinkles by silicone replicas and improved hydration, elasticity and brightness of eyelid skin [5] [6]. The current investigation in Protocol \#1 demonstrated that the use of growth factors can have a beneficial impacton improving the overall condition of skin compared to a placebo split-face treatment. By VISIA Analysis, the use of Lifeline resulted in reduction of pigmented spots and UV light damage including more effective wrinkle effacement and textural smoothness after three months than that observed on the placebo side throughout the study (Figures 1-3; Figure 9). Greater improvement in the reduction of number of porphyrin and pore spots can be anticipated with longer usage of growth factors than the three months of treatment as adhered to in this study. 
Placebo versus Lifeline (VISIA Analysis)

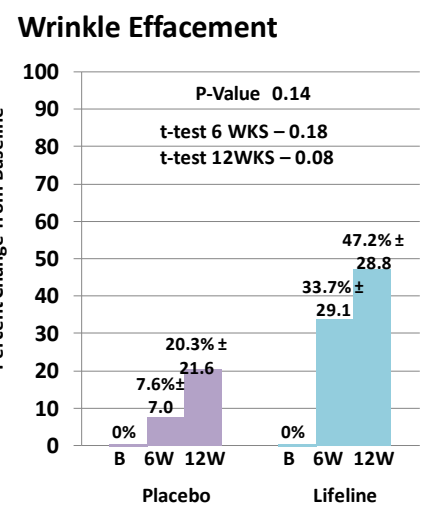
Texture Improvement

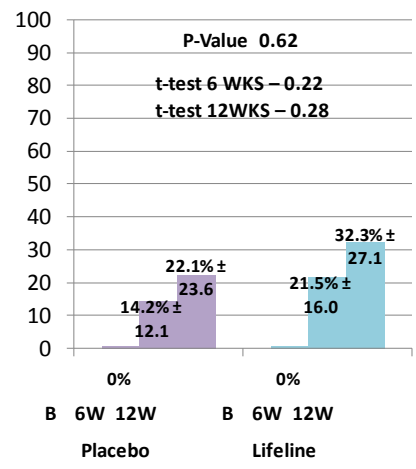

Figure 2. VISIA Analysis of average percent change of absolute values from baseline values (percent $\pm \mathrm{SD}$ ) in improvement of wrinkles and texture on placebo vs. Lifeline sides of face at six and twelve weeks.

\section{Placebo versus Lifeline (VISIA Analysis)}

\section{Pore Reduction}

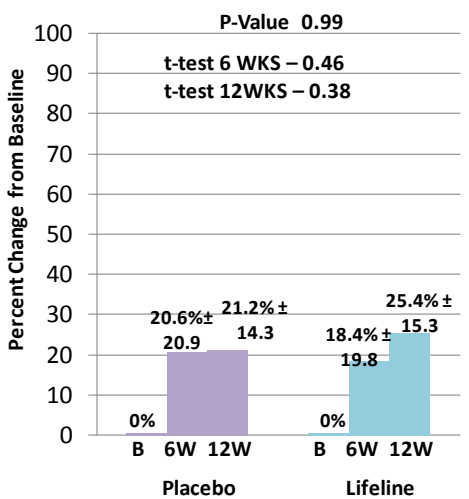

Porphyrins (Bacteria) Reduction

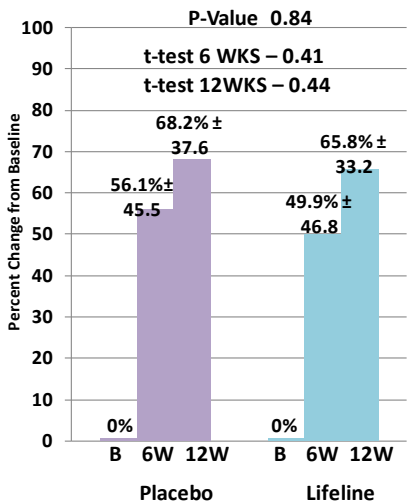

Figure 3. VISIA Analysis of average percent change of absolute values from baseline values (percent \pm SD) in reduction of pore size spots and porphyrin spots on placebo vs. Lifeline sides of face at six and twelve weeks.

\section{Lifeline versus Lifeline/Microneedling(VISIA Anaylsis)}
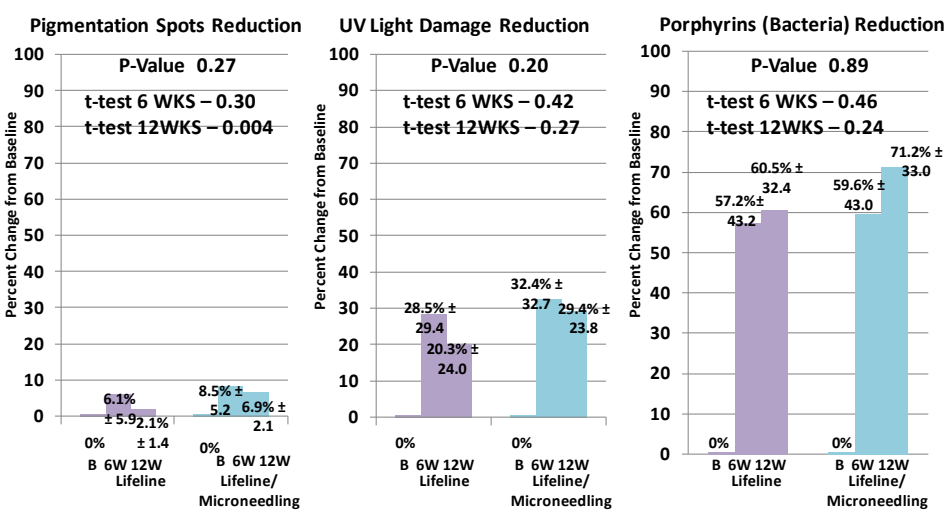

Figure 4. VISIA Analysis of average percent change of absolute values from baseline values (percent $\pm \mathrm{SD}$ ) in reduction of pigmentation spots, UV spots, and porphyrin spots on Lifeline vs. Lifeline/micro-needling sides of face at six and twelve weeks. 


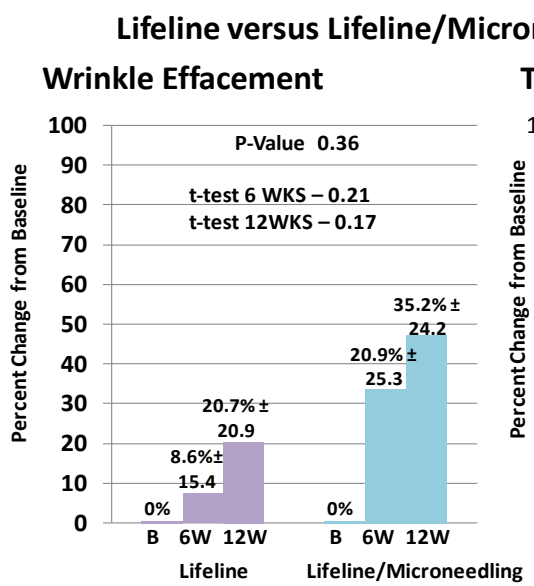

Texture Improvement

Figure 5. VISIA Analysis of average percent change of absolute values from baseline values (percent \pm SD) in improvement of wrinkles and texture on Lifeline vs. Lifeline/micro-needling sides of face at six and twelve weeks.

Lifeline versus Life line/Microneedling (VISIA Anaykis)

\section{Pore Reduction}

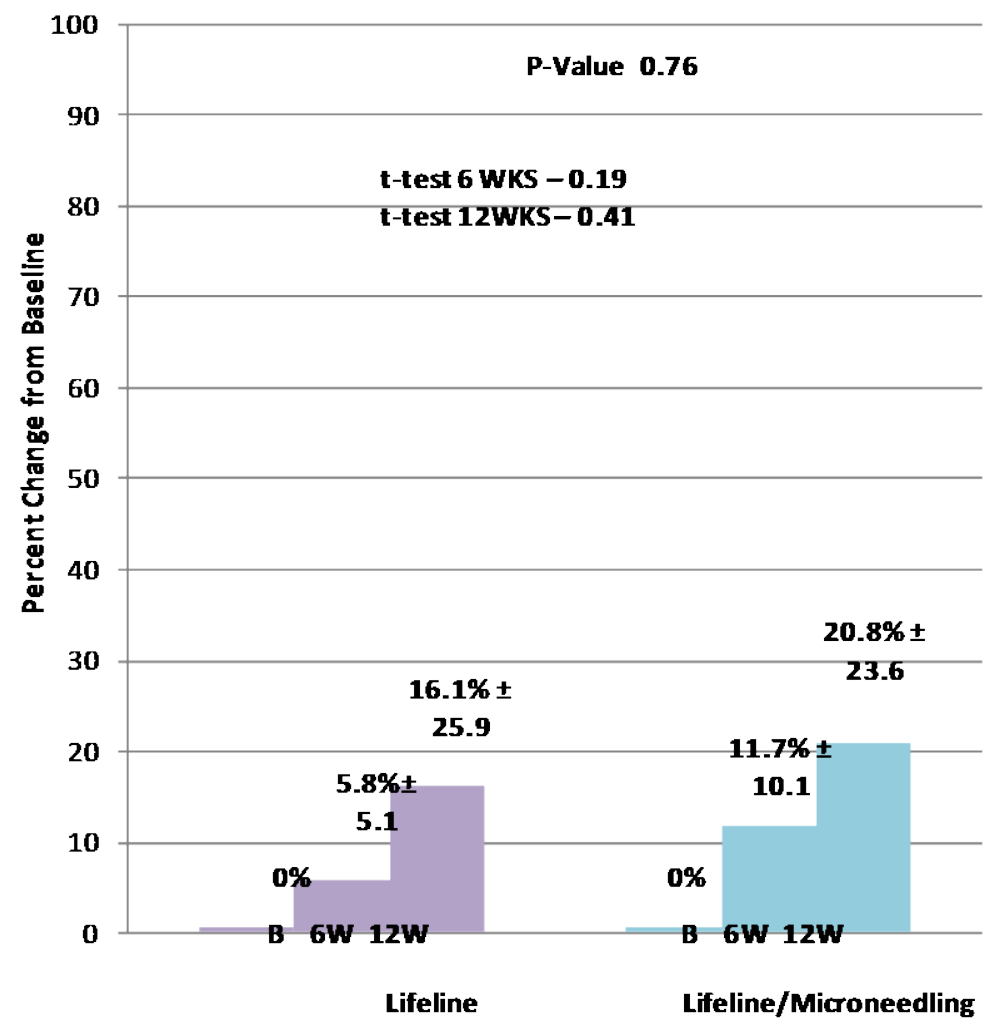

Figure 6. VISIA Analysis of average percent change of absolute values from baseline values (percent $\pm \mathrm{SD}$ ) in reduction of pore size spots on Lifeline vs. Lifeline/micro-needling sides of face at six and twelve weeks.

In Protocol \#2, VISIA Analyses of skin, treated with Lifeline/micro-needling, demonstrated marked improvement in the reduction of pigmentations, UV light damage, porphyrin spots, wrinkles and textural smoothness over that observed in subjects treated with Lifeline alone for three months. The combined use of micro-needling and 


\section{Lifeline versus Lifeline/Microneedling (DermaLab - Cortex Analysis)}

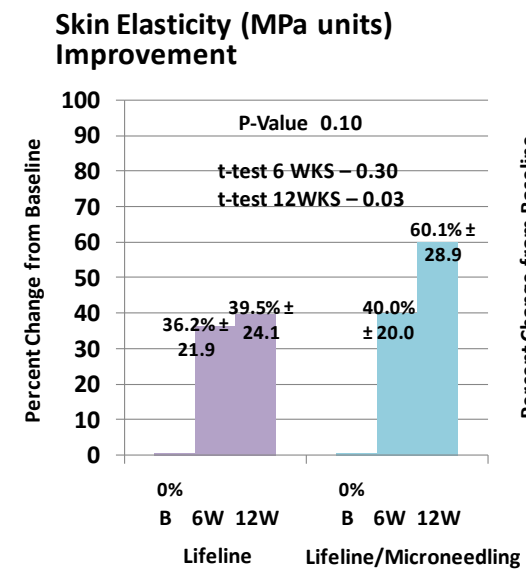

Hydration ( $\mu$ s units) Improvement

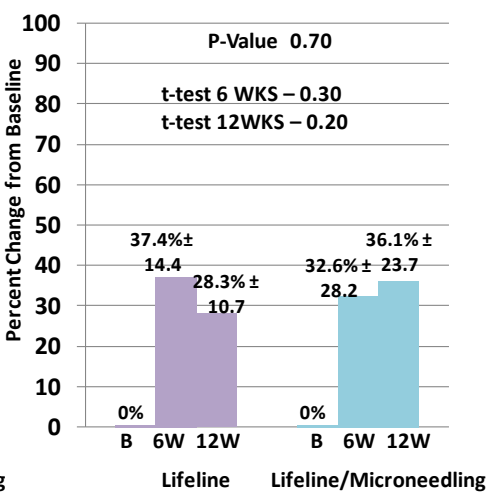

Figure 7. Cortex Analysis of average percent change of absolute values from baseline values (percent $\pm \mathrm{SD}$ ) in improvement in skin elasticity (MPa units) and hydration ( $\mu$ s units) on Lifeline vs. Lifeline/micro-needling sides of face at six and twelve weeks.

\section{Lifeline versus Lifeline/Microneedling \\ (DermaLab - Cortex Analysis)}
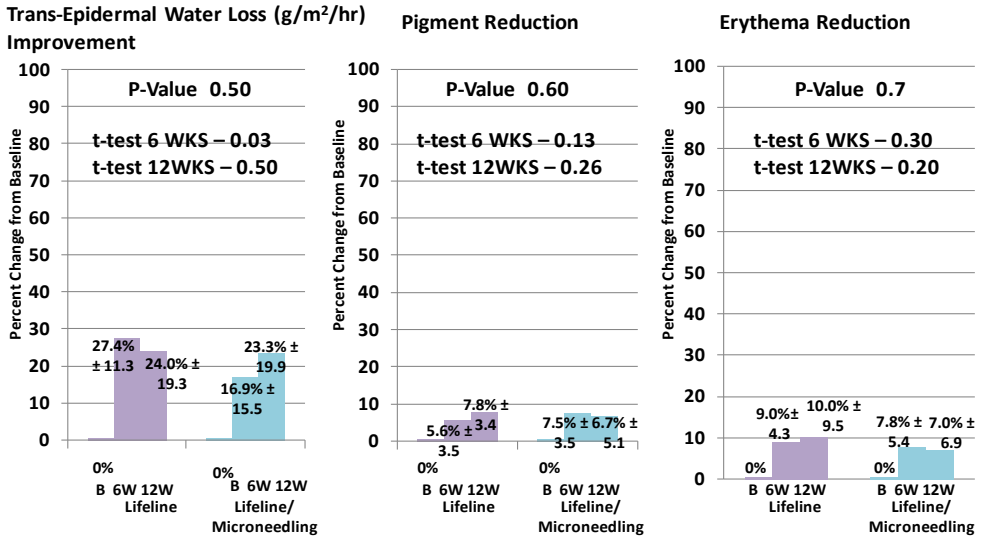

Figure 8. Cortex Analysis of average percent change of absolute values from baseline values (percent \pm SD) in reduction in transdermal water loss $\left(\mathrm{g} / \mathrm{m}^{2} / \mathrm{hr}\right)$, pigmentation, and erythema on Lifeline vs. Lifeline/micro-needling sides of face at six and twelve weeks.

Lifeline provided no additional improvements in pore size and number than that observed at three months after Lifeline only (Figures 4-6; Figure 10). Cortex Analyses of skin, receiving Lifeline/micro-needling, demonstrated marked improvement in skin elasticity and hydration over that observed in skin treated with Lifeline only for three months (Figure 7). Lifeline's beneficial effects on re-establishing and maintaining the protective lipid layers in the epidermis to reduce trans-epidermal water loss, as well as pigment and erythema presence, was not markedly enhanced with the addition of micro-needling to the treatment regimen (Figure 8).

No adverse reactions to topical treatments with growth factors, non-cosmeceutical moisturizers, or microneedling were observed or reported throughout the duration of study. The occasional subject experienced transient irritation with the usage of growth factors in the first week that resolved itself within a few days and did not alter the treatment algorithm. While the long-term use of growth factors raises the potential of stimulating the growth of skin cancers [7]-[9], there has been no reported documentation in humans of such occurrences 

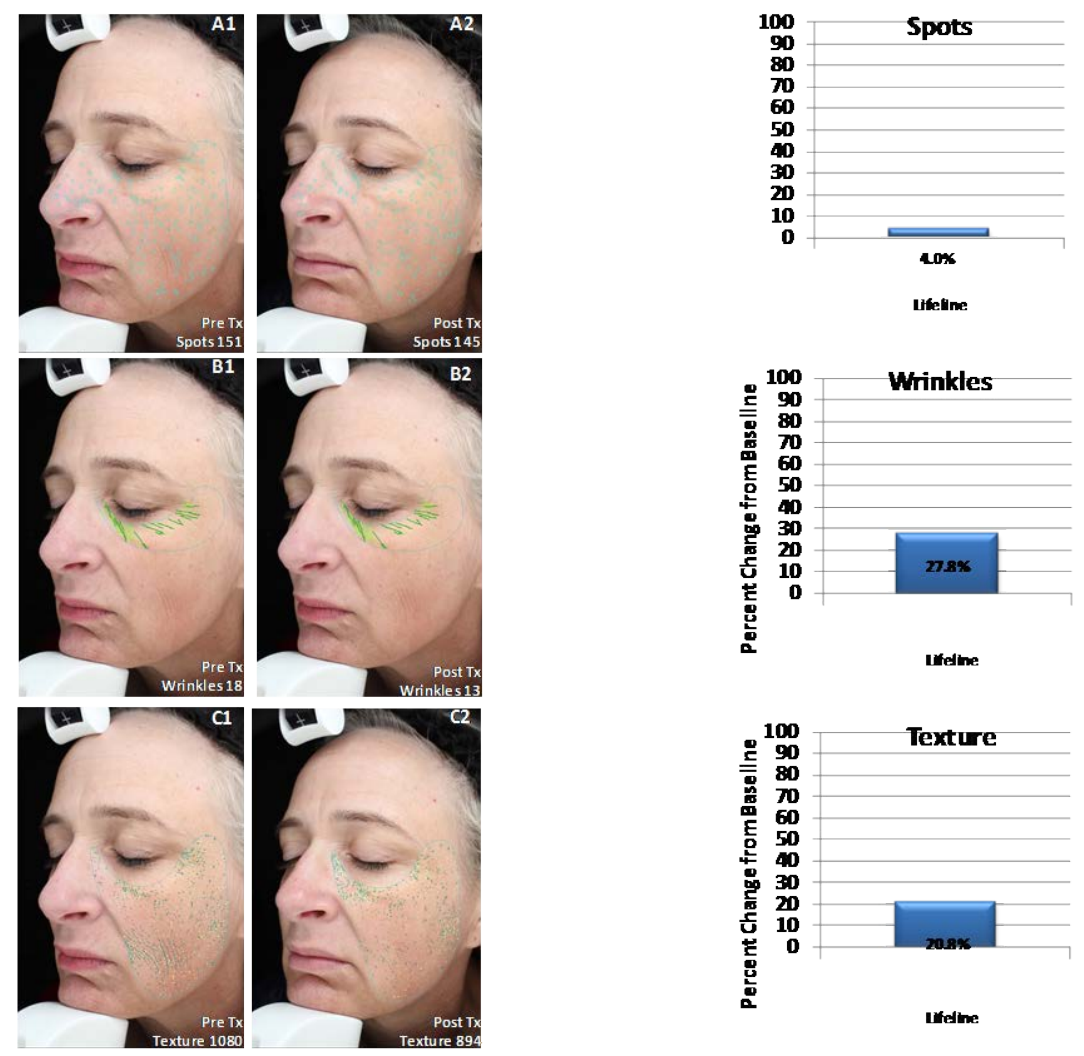

(a)
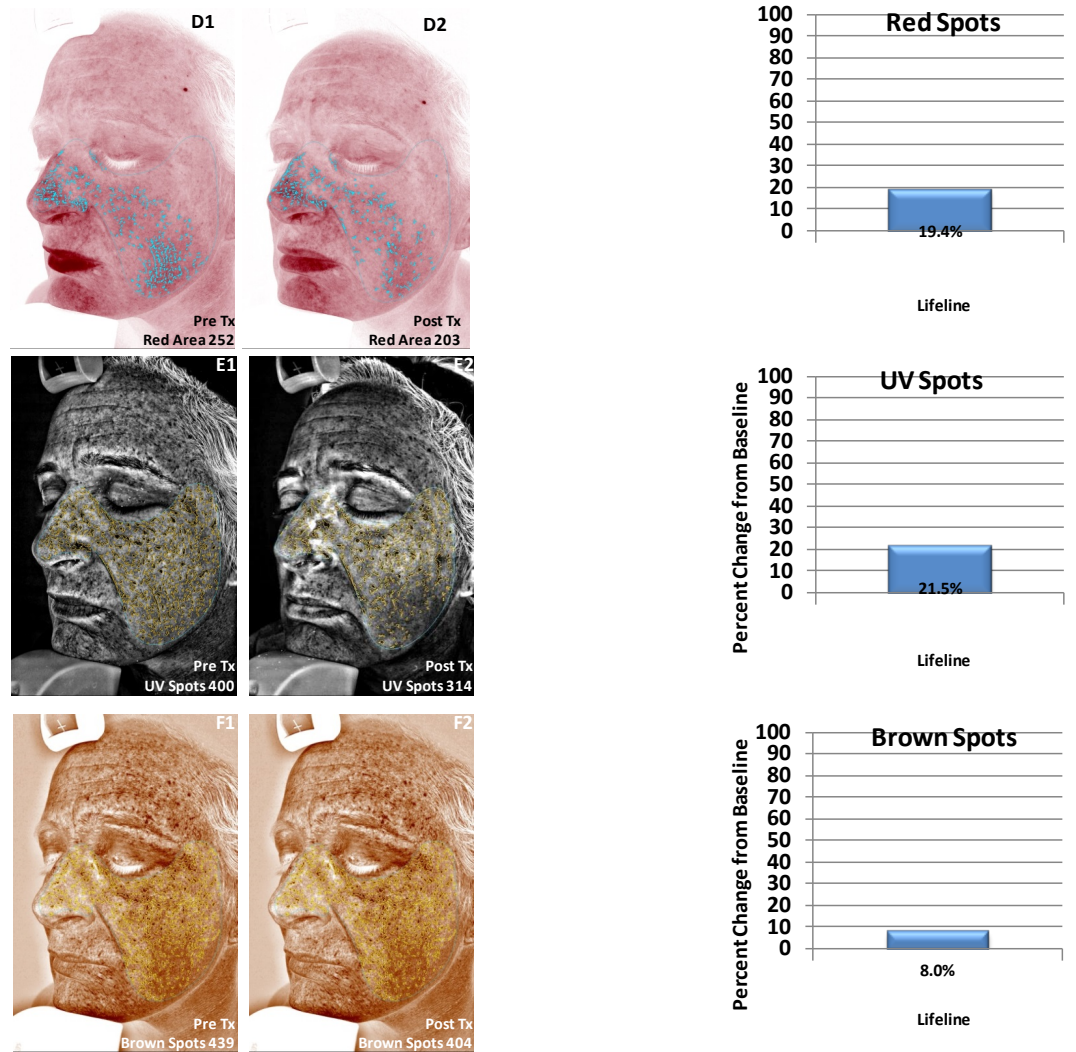

(b) 

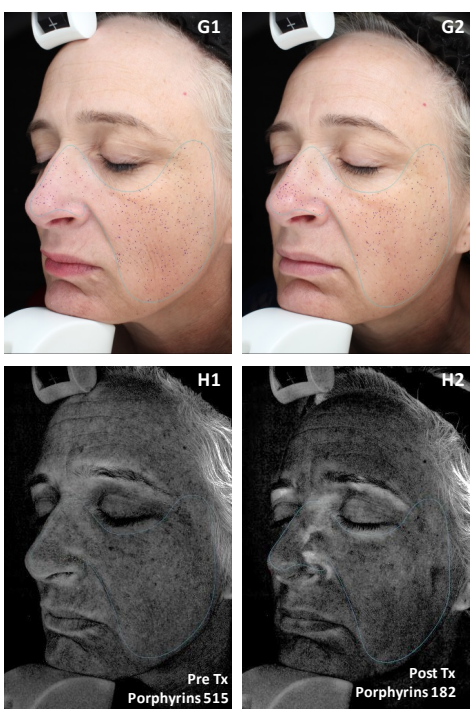
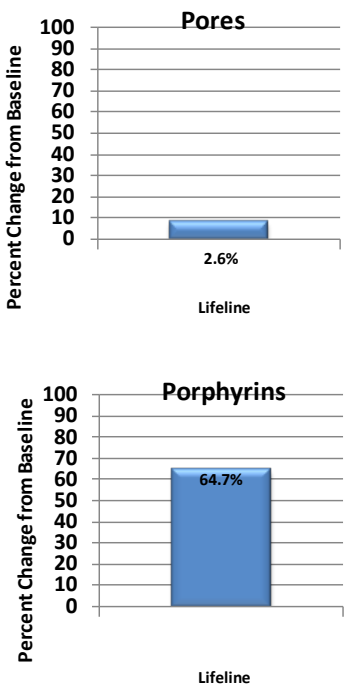

(c)

Figure 9. A typical example of improved results in reduction of pigmentation spots, wrinkles, texture, pores spots, UV spots, brown spots, red spots, and porphyrin spots on the Lifeline-treated side of a subject enrolled in Protocol \#1. By VISIA Analysis, the results are expressed both in absolute values in the bottom right corner of photographs at baseline and 3 months and in percent change from baseline values to 3 months in the accompanying graphs. On the placebo-treated side (not shown), this subject demonstrated minimal differences in the absolute values of all features from baseline to 3 months.
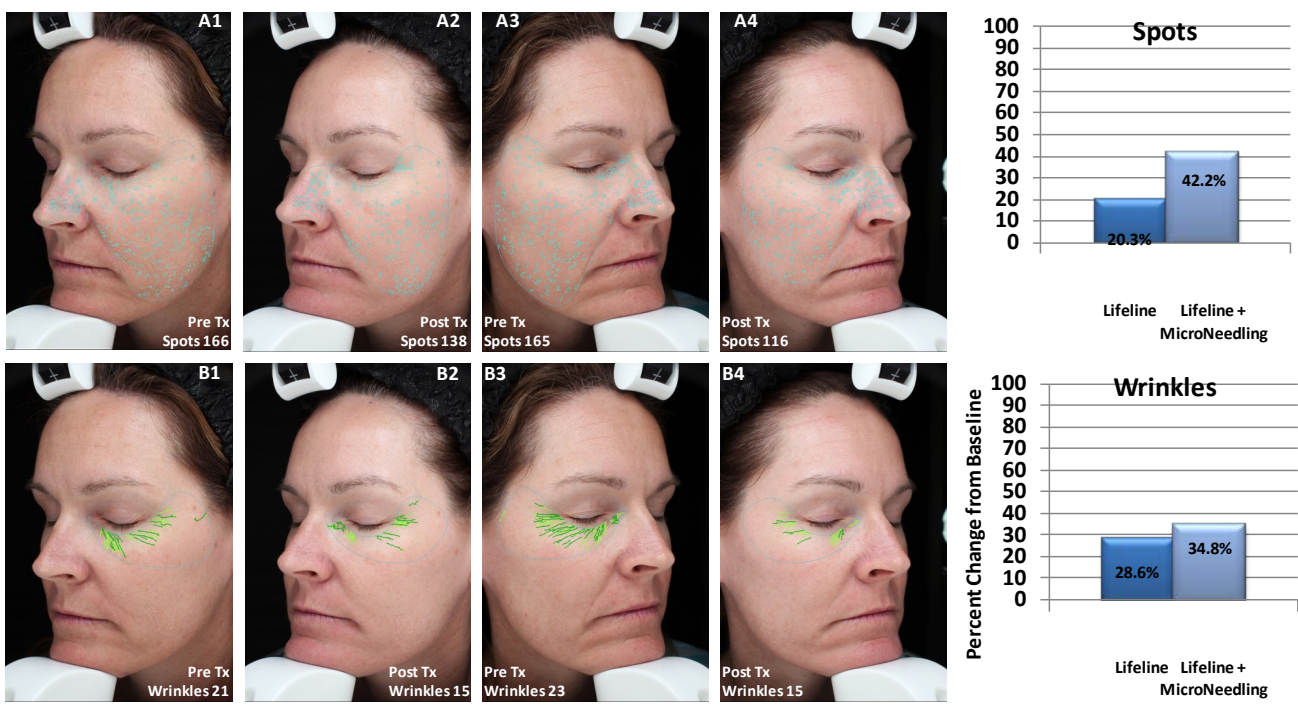

Lifeline Lifeline +

MicroNeedling
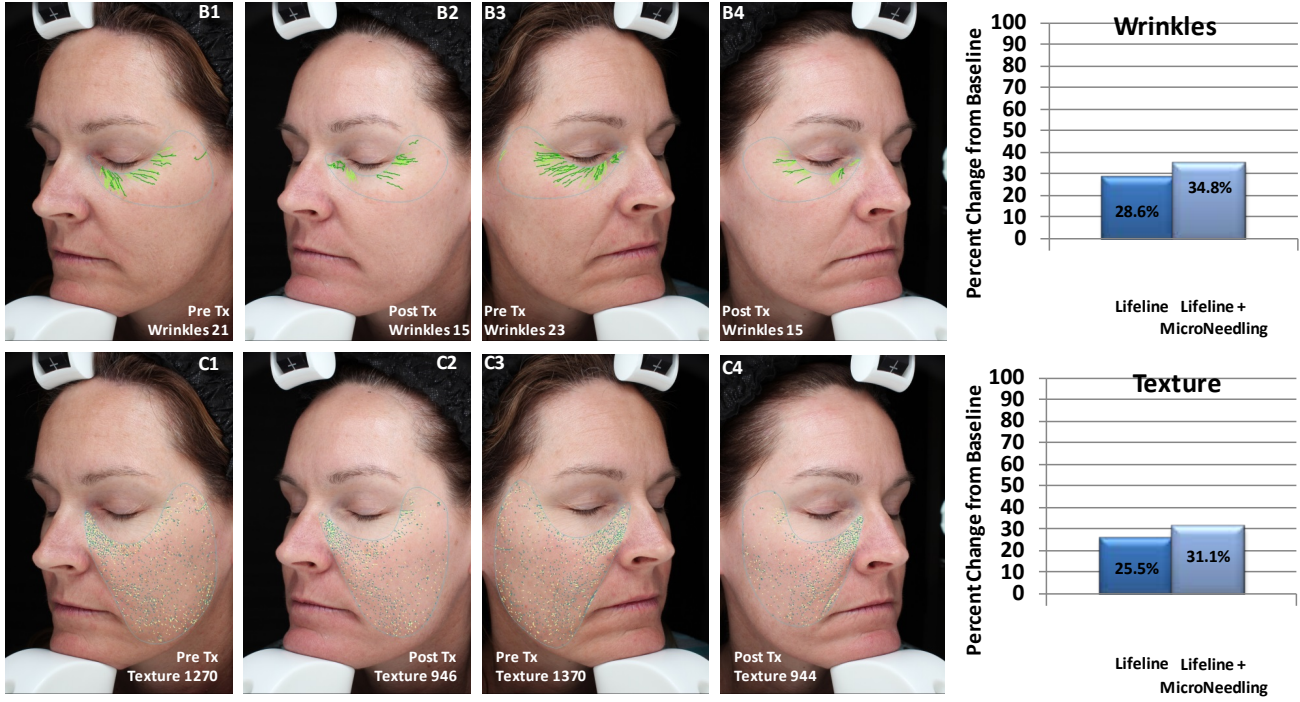

(a) 

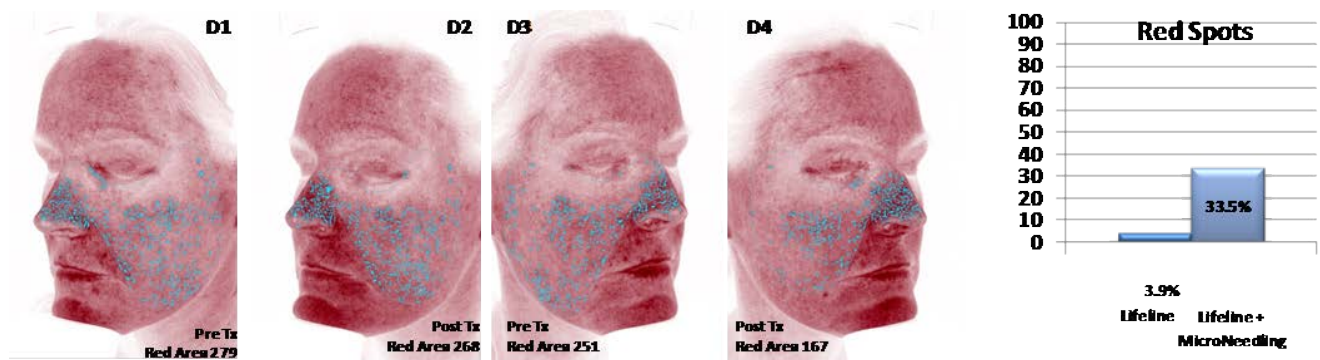

Red Area 167
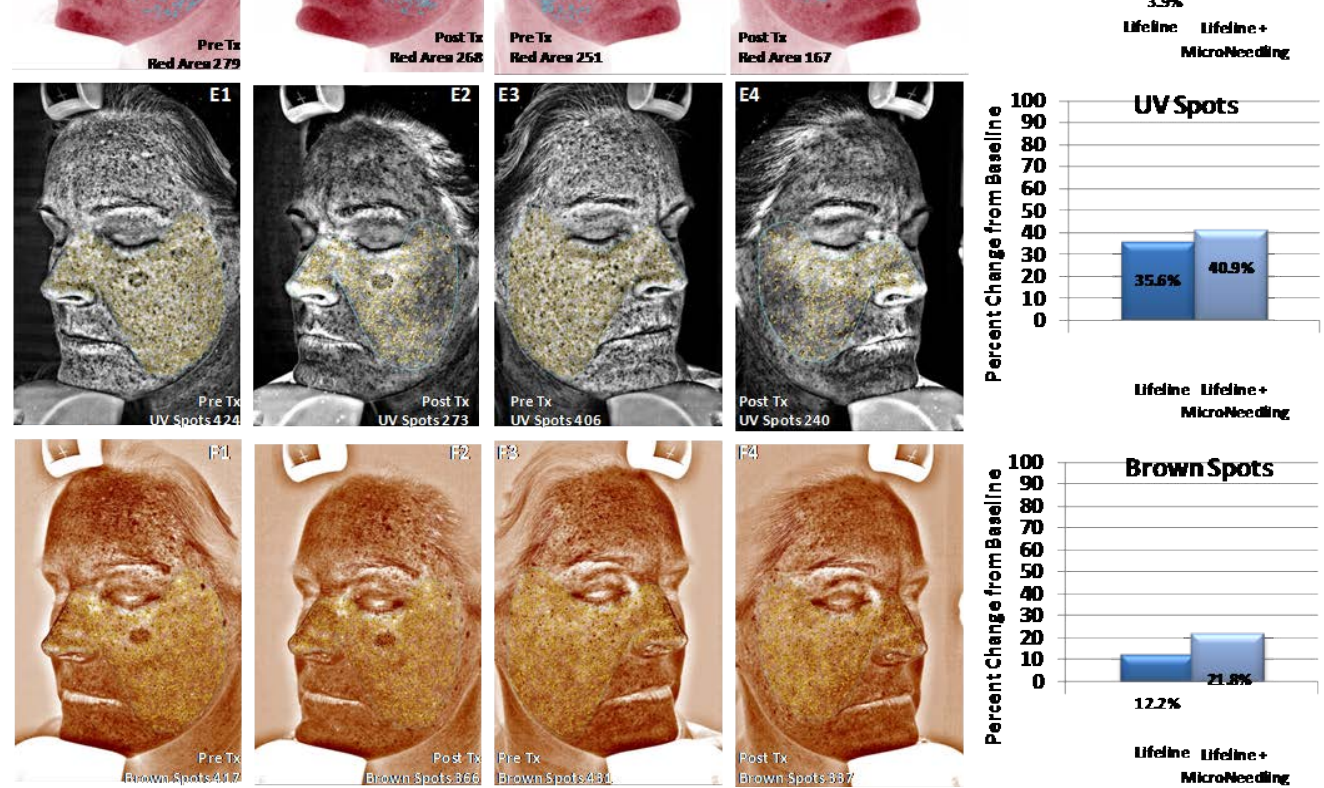

(b)
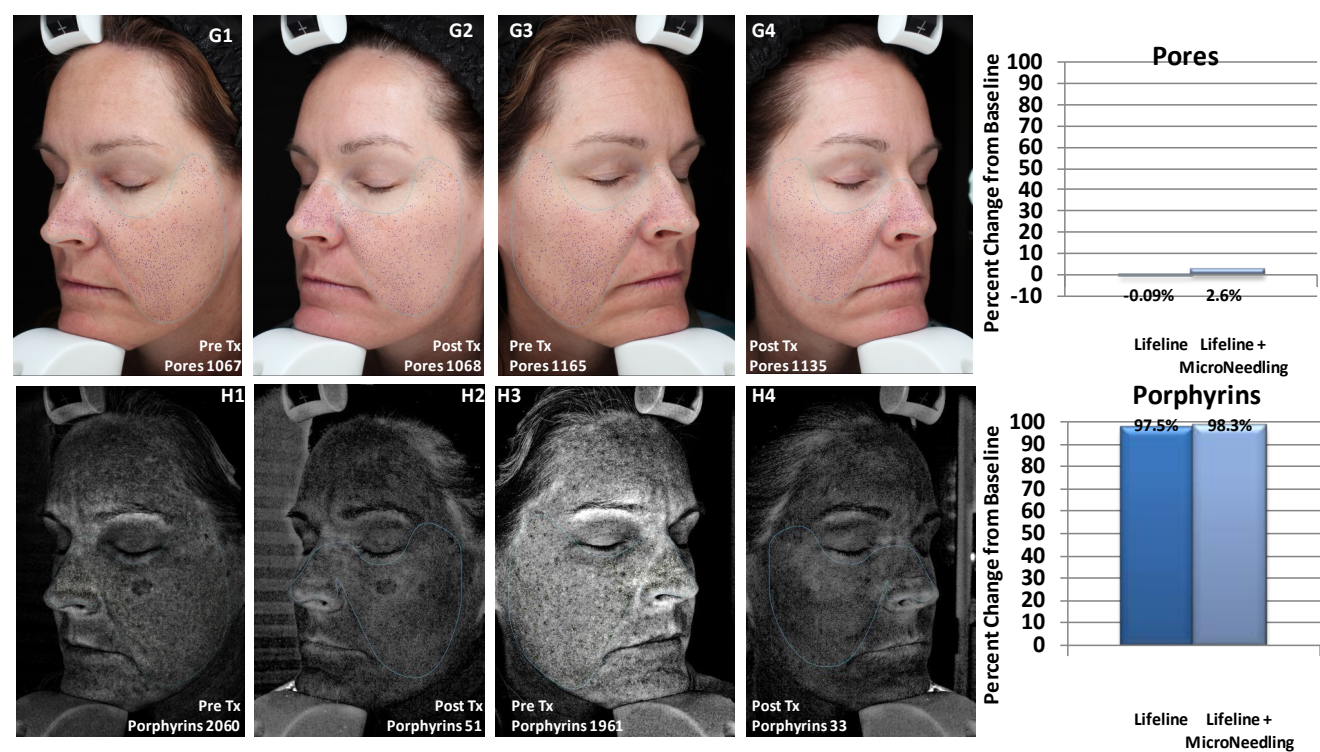

(c)

Figure 10. A typical example of improved results in reduction of pigmented spots, wrinkles, texture, red spots, UV spots, pore size spots and porphyrin spots on the Lifeline-treated left side of face (Panel A) and on Lifeline/micro-needling-treated right side of face (Panel B) that are expressed in absolute values by VISIA Analysis from baseline to 3 months. Panel $\mathrm{C}$ compares the Lifeline percent improvement to Lifeline/micro-needling from baseline to 3 months for pigmented spots, wrinkles, texture, red spots, UV spots and brown spot. The addition of micro-needling to Lifeline treatment did not increase the beneficial effects observed from Lifeline for pores and porphyrin spots after 3 months of treatment. 
with the use of this product. Although no statistical significance was able to be calculated for each of the parameters due to wide ranges of measurements at each study interval, there was a definite trend toward improved results with Lifeline treatment over placebo and after micro-needling over Lifeline treatment. A larger number of subjects, a longer usage products and micro-needling, and longer follow-up periods will be needed to evaluate the safety and efficacy of this product alone or when combined with micro-needling.

\section{Conclusion}

In summary, the use of growth factor containing creams have a salutary effect on photo-aging skin and can be enhanced with the addition of micro-needling without introducing significant side-effects or complications. Further investigations with a larger cohort of subjects for longer treatment periods will be needed to confirm and extend these initial findings.

\section{References}

[1] Babu, M. and Wells, A. (2001) Dermal-Epidermal Communication in Wound Healing. Wounds, 13, 183-189.

[2] Wermeling, D.P., Banks, S.L., Hudson, D.A., et al. (2008) Microneedles Permit Transdermal Delivery of Skin-Impermeant Medications to Humans. Proceedings of the National Academy of Sciences of the United States of America, 105, 2058-2063. http://dx.doi.org/10.1073/pnas.0710355105

[3] Prausnitz, M.R., Mitragotri, S. and Langer, R. (2004) Current Status and Future Potential of Transdermal Drug Delivery. Nature Reviews Drug Discovery, 3, 115-124. http://dx.doi.org/10.1038/nrd1304

[4] Fernandes, D. (2002) Percutaneous Collagen Induction: An Alternative to Laser Resurfacing. Aesthetic Surgery Journal, 22, 315-317. http://dx.doi.org/10.1067/maj.2002.126195

[5] Lifeline Skin Care, Inc. (2013) Controlled Usage Study to Evaluate Efficacy of an Anti-Aging Product. Study Conducted by AMA Laboratories, Inc., New York.

[6] Lifeline Skin Care, Inc. (2013) Evaluation of the Efficacy of a Topical Skin Care Regimen to Improve Skin Condition. Study Conducted by Bioscreen Testing Services, Inc., Phoenix.

[7] Liu, B., Earl, H.M., Baban, D., et al. (1995) Melanoma Cell Lines Express VEGF Receptor KDR and Respond to Exogenously Added VEGF. Biochemical and Biophysical Research Communications, 217, 721-727. http://dx.doi.org/10.1006/bbrc.1995.2832

[8] Lazar-Molnar, E., Hegyesi, H., et al. (2000) Autocrine and Paracrine Regulation by Cytokines and Growth Factors in Melanoma. Cytokine, 12, 547-554. http://dx.doi.org/10.1006/cyto.1999.0614

[9] Draelos, Z.D. (2007) Exploring the Pitfalls in Clinical Cosmeceutical Research. Journal of Cosmetic Dermatology, 20, $556-558$. 